\title{
Totally autologous Ross procedure
}

Jen-Ping Chang, MD, Chiung-Lun Kao, MD, and

Ming-Jang Hsieh, MD, Taiwan, Republic of China

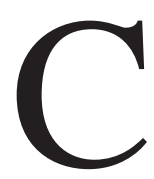

urrently, a pulmonary homograft is preferred worldwide as the right ventricular outflow tract (RVOT) substitute in the Ross procedure. The lack of pulmonary homograft limits the use of the Ross procedure in many parts of the world. To circumvent the problem, several alternatives have been reported. ${ }^{1-6}$ The autologous pedicled pericardial valved conduit (APPVC) has been reported as a modification of the Rastelli procedure with the expectation of potential conduit growth and low incidences of excessive peel formation and thrombosis. ${ }^{7}$ In this article we present a case of RVOT reconstruction with APPVC as the totally autologous Ross procedure.

\section{Clinical Summary}

The patient was a 41-year-old man with a history of completely treated streptococcal infective endocarditis 3 years previously. The chest radiograph revealed moderate cardiomegaly, and the electrocardiogram showed left ventricular hypertrophy. Echocardiography demonstrated severe aortic regurgitation and a dilated left ventricle.

The operation was performed with moderate hypothermic cardiopulmonary bypass and antegrade cardioplegia. The pericardium was incised along the right mediastinal margin to make a pedicled rectangular flap. The flap was rolled up and sutured with interrupted absorbable stitches to construct a conduit with a diameter of $25 \mathrm{~mm}$. The caudal margin of the conduit was folded upward for $10 \mathrm{~mm}$. The upper margin of the inner layer was secured to the

From the Division of Thoracic and Cardiovascular Surgery, Chang Gung Memorial Hospital at Kaohsiung, Taiwan, Republic of China.

Received for publication Oct 10, 2001; accepted for publication Jan 7, 2002.

Address for reprints: Chiung-Lun Kao, MD, Division of Thoracic and Cardiovascular Surgery, Chang Gung Memorial Hospital at Kaohsiung, 123, Ta-Pei Rd, Niao Sung Hsiang, Kaohsiung Hsien, Taiwan, Republic of China (E-mail: c9112772@adm.cgmh.org.tw).

J Thorac Cardiovasc Surg 2002;124:194-5

Copyright (C) 2002 by The American Association for Thoracic Surgery

$0022-5223 / 2002 \$ 35.00+0 \quad \mathbf{1 2 / 5 4 / 1 2 3 2 5 4}$

doi: $10.1067 / \mathrm{mtc} .2002 .123254$

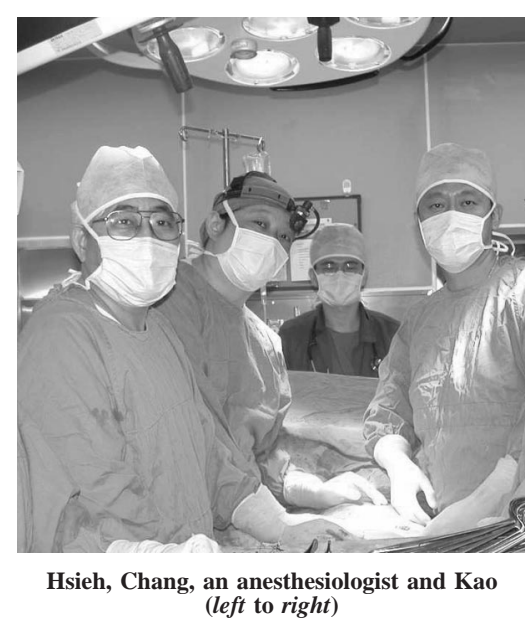

outer layer at three equidistant points, to become the future valve commissures (Figure 1).

The Ross procedure was completed in the usual manner. The APPVC was then used for the RVOT reconstruction. With 5-0 absorbable monofilament running sutures, the distal end of the conduit was anastomosed to the pulmonary artery. The proximal anastomosis was created with the interposition of a short segment of autologous aortic tube between the conduit and the RVOT, because this required less suturing and no prosthetic conduit had a subvalvular or anterior hood segment (Figure 2).

The postoperative course was uneventful. At 36 months after the operation, the patient was well. Serial echocardiographic follow-up revealed stable valvular condition with mild pulmonary stenosis (the Doppler echocardiographic mean gradient was 22 $\mathrm{mm} \mathrm{Hg}$ ) and regurgitation. The aortic valve was satisfactory (trivial aortic regurgitation without any pressure gradient).

\section{Discussion}

Currently, indications for the Ross procedure are expanding. Autografts show excellent longevity, clear hemodynamic superiority, infection resistance, and freedom from anticoagulation. These characteristics are attractive, even though the procedure is complex and time-consuming. A pulmonary homograft is accepted worldwide as the material most useful for the RVOT reconstruction in Ross procedure. Although a pulmonary homograft is preferred and lasts longer in the pulmonary position, replacement nevertheless will be needed. Furthermore, developing countries where homografts are not available do not currently have an acceptable alternative. To circumvent those problems, numerous types of conduits have been developed, each with its own advantages and disadvantages, to connect the right ventricle to the pulmonary artery.

Most of these alternatives are composite grafts of autologous tissue and artificial materials (polytetrafluoroethylene) or porcine bioprostheses. ${ }^{1-6}$ They have their own limitations with respect to growth potential or valvular competency. In contrast, the APPVC seems to circumvent these problems in most cases.

Our technique does raise several issues. First, the use of autologous pedicled pericardial conduit is important. It has been reported that RVOT reconstruction with autologous pedicled pericardium is useful to prevent late stenosis, because the pedicled pericardium is more pliable and less fibrotic. ${ }^{8}$ Therefore this con- 


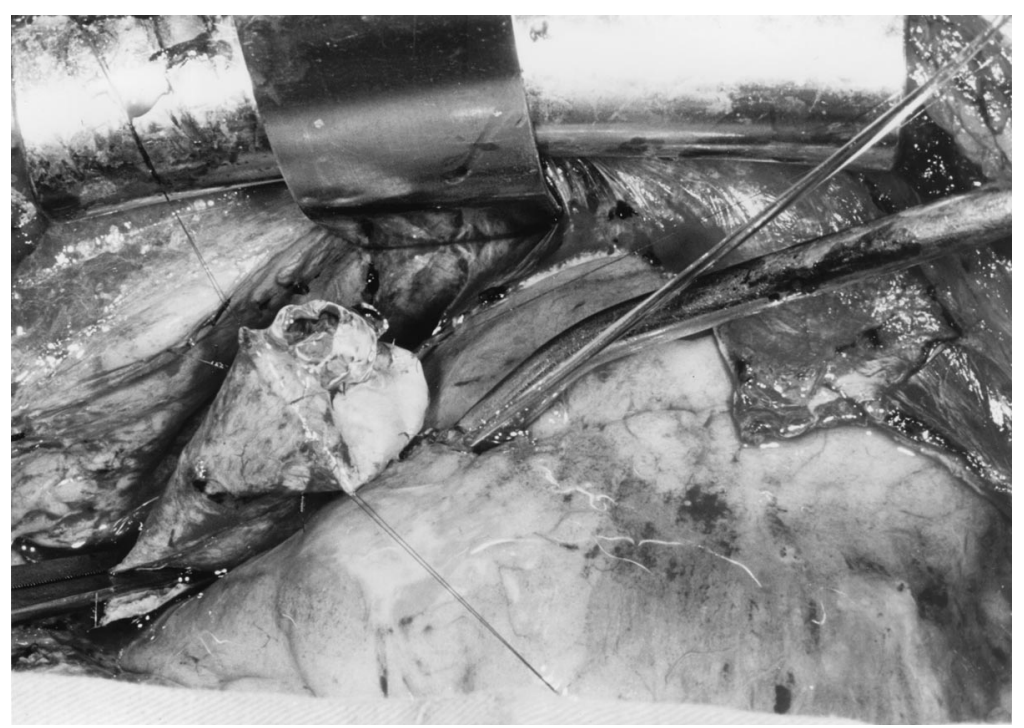

Figure 1. Competent APPVC.

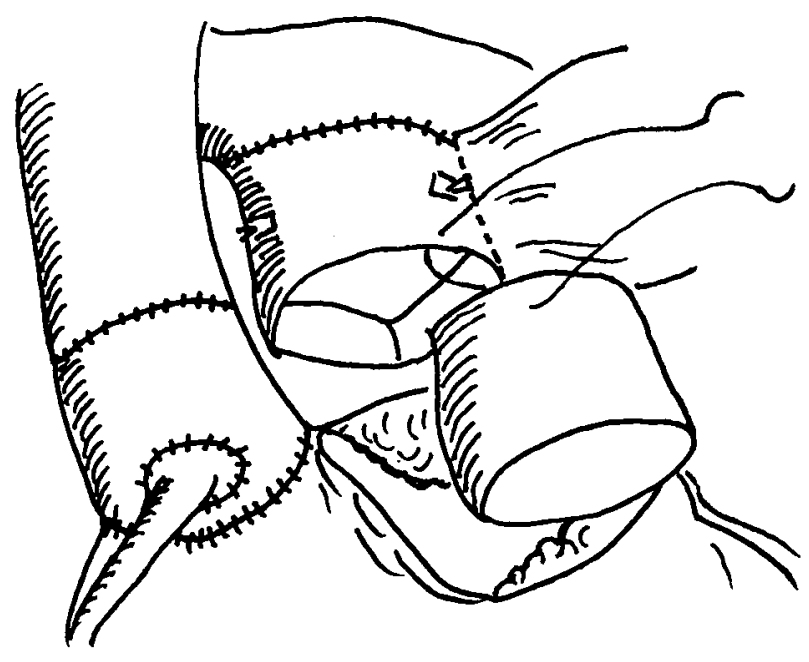

Figure 2. Short segment of autologous aortic tube interposed between conduit and right ventricle.

duit is expected to have good longevity. Another issue is the role of the pericardial tricuspid valve. Usually, a tricuspid valve is considered more effective than a monocuspid valve in preventing pulmonary regurgitation. To avoid cusp adhesion to the conduit wall, we used no prosthetic material. Therefore the pericardial flap should be as long as possible, and potential growth of the valved conduit may be expected. ${ }^{7}$

In conclusion, we emphasize that the APPVC may be a good alternative for RVOT reconstruction in the Ross procedure. It provides the expectation of a functioning tricuspid pericardial valve, prevention of late stenosis, and potential conduit growth.

\section{References}

1. Urrea MS, Herrena V, Rey A, Vargus J. Ross operation using a bovine bioprosthetic valve with autologous pericardial conduit in the pulmonary position. Tex Heart Inst J. 1993;20:271-4.

2. DeLeon SY, Quinones JA, Miles RH, Hofstra J, Bell TJ, Fisher EA, et al. Use of the native aortic valve as the pulmonary valve in the Ross procedure. Ann Thorac Surg. 1995;59:1007-10.

3. Morishita K, Abe T, Fukada J, Sato H, Shiiku C. Alternative to reconstruction of the pulmonary outflow tract in the Ross procedure. Ann Thorac Surg. 1998;66:549-50.

4. Yamagishi M, Emmoto T, Wada Y, Oka T. Pulmonary reconstruction in the Ross procedure: combined autologous aortic and polytetrafluoroethylene valve. J Thorac Cardiovasc Surg. 1998;116:1076-7.

5. Tabayashi K, Tanaka Y, Endo M, Sai S, Masuda S, Sadahiro M. Right ventricular outflow reconstruction with nonsutured pedicled autologous pericardium. J Thorac Cardiovasc Surg. 2001;121:1203-5.

6. Marianeschi SM, Iacona GM, Seddio F, Abella RF, Condoluci C, Cipriani A, et al. Shelhigh No-react porcine pulmonic valve conduit: a new alternative to the homograft. Ann Thorac Surg. 2001;71:619-23.

7. Iemura J, Oku H, Otaki M, Kitayama H, Matsumoto T. Reconstruction of right ventricular outflow tract by pedicled pericardial valved conduit. Ann Thorac Surg. 1997;64:1849-51.

8. Sato K, Iguchi A, Tanaka Y, Hata M, Sadahiro M, Yokoyama H, et al. Pedicled pericardial flap for pulmonary artery in adult dogs. Jpn J Thorac Cardiovasc Surg. 2000;48:211-6. 